\title{
Party Panel: the architecture of a semi-panel study to flexibly map determinants of nightlife risk behaviors and inform prevention efforts
}

Gjalt-Jorn Ygram Peters \& Judith Noijen

This document is the full text of the manuscript "Party Panel: the architecture of a semi-panel study to flexibly map determinants of nightlife risk behaviors and inform prevention efforts" that has been submitted as a book chapter (and is currently under review).

The most current version of this manuscript is hosted at PsyArXiv at https://psyarxiv.com/akpmx - please check that URL before citing this article.
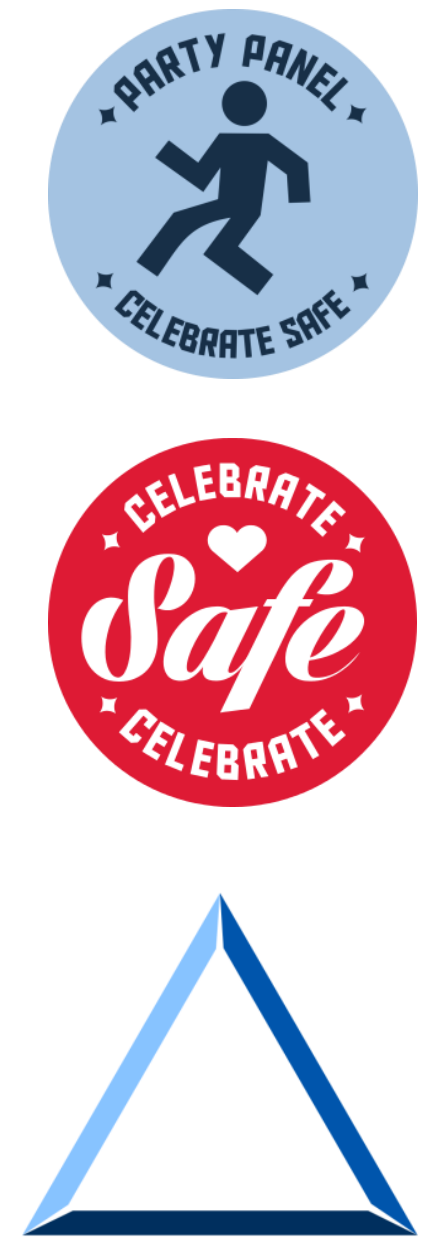

Academy of Behavior Change

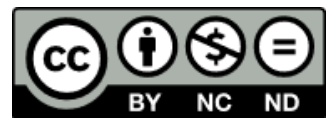


risk behaviors and inform prevention efforts

Participation in nightlife activities, like other leisure activities, conveys numerous benefits

(Tinsley \& Eldredge, 1995). However, leisure activities can also carry risk. For example, physical

exercise can result in hyponatremia (Montain, Sawka, \& Wenger, 2001), go-karting may result in injuries of the spinal column (Eker, Lieshout, Hartog, \& Schipper, 2010), and sedentary leisure activities such as reading books or watching television have been associated to obesity and related negative health consequences (Hu, Li, \& Colditz, 2003; Martinez-Gonzalez, Alfredo Martinez, Hu, Gibney, \& Kearney, 1999). Nightlife participation is no exception (Bellis, Hughes, McVeigh, Thomson, \& Luke, 2005) and is associated to, for example, increased risk of traffic accidents (e.g. Calafat \& Juan, 2004) and involvement in (often alcohol-related) violence (Hughes, Anderson, Morleo, \& Bellis, 2007). Therefore, measures to minimize such risks are warranted.

Public health interventions can be cost-effective means of reducing health risks (Masters,

Anwar, Collins, Cookson, \& Capewell, 2017), but developing an effective behavior change intervention is no straightforward affair (Glanz \& Bishop, 2010; Kok, 2016). Health risks are often determined by both environmental and psychological factors (for a broader overview, see e.g. Bartholomew Eldredge et al., 2016), and successful intervention requires first mapping these so-called determinants (G.-J.Y. Peters, 2014) before fitting behavior change methods can be selected (Kok, 2014; Kok et al., 2016). Both designing a determinant study and analyzing the data (Crutzen, Peters, \& Noijen, 2017; G.-J.Y. Peters, 2014) and selecting and applying appropriate behavior change methods 
available (Kok, 2016). This limited availability means that interventions are often developed without a systematic approach and without leveraging behavior change theory and evidence (Gjalt-Jorn Ygram Peters, Ruiter, \& Kok, 2014; Schaafsma, Stoffelen, Kok, \& Curfs, 2013). behavior change interventions to reduce nightlife risks, while on the other hand, the behavior change expertise to develop such interventions is not always available to the relevant prevention organizations. Although a number of initiatives exist to monitor nightlife-related risk behaviors such as patterns in substance use (e.g. Van Laar et al., 2016), these focus on people's behavior, not on the determinants of those behaviors. As such, they cannot inform intervention development (Crutzen et al., 2017; Gjalt-Jorn Ygram Peters \& Crutzen, 2018). One exception is a yearly multi-method panel study in the Amsterdam region where key actors in the Amsterdam nightlife are surveyed to monitor trends (Nabben, Luijk, Benschop, \& Korf, 2017). Being partly qualitative, this report can sometimes provide insight into potentially relevant beliefs. However, such outcomes are not systematically verified quantitatively. Thus, in the Netherlands, no systematic efforts are in place to inform behavior change interventions aiming to reduce nightlife-related risks.

While in the ideal situation, behavior change would be recognized as a protected profession and agencies tasked with prevention would employ such behavior change experts (see section "Basics of Behavior Change" below), this scenario has not yet been realized in the Netherlands. Until such time, the Netherlands (and other countries facing similar problems) can benefit from a structural collaboration between prevention professionals and behavior change experts. In the current contribution, we describe how we designed the infrastructure for such structural collaboration. We aim to do this in such a way that this solution can relatively easily be adopted in other contexts where 
In acknowledgement of the risks inherent in nightlife, in 2014, organizations from the Dutch private and public sectors together realized a public private partnership to advance nightlife prevention efforts (for more reflection on the value and challenges associated to such 'assemblages', see van Liempt, 2015). This partnership took the form of the Celebrate Safe campaign, a campaign that had previously been developed by ID\&T, a Dutch organizer of dance events and related activities, and was led by Jellinek Prevention, a department of Dutch healthcare association Arkin. A prevention professional from Jellinek Prevention (author JN) leads the project committee, which also consists of a representative of ID\&T, a project manager familiar with Dutch nightlife organizations, a representative of an organization specialized in medical treatment and prevention of nightlife accidents called Educare, and a representative (author GJYP) of both Greater Good, an organization specializing in behavior change, and the methodology and statistics department at a university (the Dutch Open University).

Celebrate Safe is a multifaceted project. The public face consists of the communication with nightlife patrons. This communication takes the form of physical banners and posters as well as social media posts. The physical communications mostly just list ten 'pillars' of the public face of the Celebrate Safe campaign: ten brief guidelines for responsible nightlife enjoyment, such as "Take your time to chill", "Think for yourself, care about others", and "Using alcohol or other drugs is never without risk". Sometimes, specific communications are added to address topical developments. The social media posts are tailored to address specific risks relating to, for example, weather circumstances or trends on the drug market. To enable this public facet of Celebrate Safe, a system is used where event organizers and venues can apply for ambassadorship. Ambassadors receive nightlife risk management information and are allowed to communicate their Celebrate Safe ambassadorship in communications with governmental bodies. In return, they produce and deploy the physical communication materials and share Celebrate Safe social media messages. 

ambassadorships. Second, securing and maintaining widespread support for the Celebrate Safe campaign among ambassadors, other private organizations such as security companies and medical service providers, government bodies, and prevention organizations requires constant communication and coordination. Finally, Celebrate Safe harnesses two related initiatives developed to promote effective prevention in the Dutch nightlife. One is a committee that screens interventions for their theoretical and empirical basis and provides guidance if a submitted intervention lacks a solid basis in terms of behavior change theory and evidence. This committee allows Celebrate Safe to develop a list of plausibly effective interventions to offer to ambassadors and governmental bodies. The second is Party Panel, the focus of this paper.

\section{The Party Panel lifecycle} studies and translating the results to recommendations for effective prevention efforts. Every Party Panel wave has a lifecycle consisting of four consecutive stages that roughly follow the first three steps of the Intervention Mapping protocol for systematic development of theory- and evidence based interventions (Bartholomew Eldredge et al., 2016).

The first stage is comparable to step 1 of Intervention Mapping: the needs assessment. It starts with a nightlife-related risk issue, such as concerns about higher dosing of 3,4Methylenedioxymethamphetamine (MDMA) because of rising ecstasy pill strength, the observation that not all patrons experiencing medical problems visit the on-site first aid facilities, or a concern for hearing damage sustained in nightlife venues. This concern is narrowed down and integrated in an online survey where participants can nominate phenomena, risk behaviors, or potential problems that they think contribute to the issue at hand. This survey is then distributed amongst four groups of experts. First, peer educators of the Dutch dance-scene based peer education project Unity (a project 
initiated by Jellinek Prevention, the same organization heading the Celebrate Safe project, and now also implemented by a total of six Dutch prevention organisations: Brijder, Indigo, IrisZorg, and Novadic-Kentron); second, experts in several behavioral and health domains such as sexual health, substance use, and hearing protection; third, experts with respect to nightlife settings and organisations; and fourth, all members of the Celebrate Safe project committee. The product of this first stage is a number of potential risk behaviors and other relevant factors.

This list is then used to compile a final list of target behaviors. Ideally, only one target behavior is selected, but this is rarely enough to gain a sufficiently thorough understanding of the issue. Because in behavior change research (and therefore, intervention development), it is important to be sufficiently specific about the target behavior (Fishbein \& Ajzen, 2010; Gjalt-Jorn Ygram Peters \& Crutzen, 2017), several behaviors are often selected in this stage. Following this selection, a second online survey is created, where for each target behavior two questions are posed. Which question is posed first depends on whether the behavior is a risk behavior or a preventive behavior. For risk behaviors, participants are first asked why they think people who exhibit that behavior do so, and then, why they think people who don't exhibit that behavior, do not. For preventive behaviors, the order is the other way around, so that the first question always addresses the undesirable scenario and the second question, the desirable scenario. These two questions are repeated for every selected target behavior. In addition, participants can suggest additional questions to include in the final Party Panel questionnaire. This survey is then presented to the Unity peers. This second stage is designed to approximate a qualitative investigation into the beliefs and determinants of the target behavior(s), which is one of the tasks in step 2 of Intervention Mapping: the mapping of the determinants of the target behavior(s). The product of this second stage is a list of potential beliefs and reasons related to the target behavior(s).

The third stage in the Party Panel lifecycle is the development and deployment of the actual Party Panel questionnaire. The information gathered in the first two stages is combined with 
psychological theory and empirical evidence available in the literature to develop a questionnaire measuring the potential determinants of the target behaviors, the frequency and intensity of target behavior performance, and additional questions relating to the focal issue of that Party Panel wave (see below for more details about the questionnaire). The associated expert organizations and the Celebrate Safe ambassadors are invited to promote the questionnaire in their networks. The data are analyzed using the Confidence Interval-Based Estimation of Relevance approach (CIBER; Crutzen et al., 2017) to determine which determinants and sub-determinants (i.e. beliefs) seem the best intervention targets (for more background, see section "Basics of behavior change" below). The product of this stage is an overview of promising intervention targets (which could be considered an approximation of the matrices of change objectives that are generated as the product of the second step if the Intervention Mapping protocol is applied comprehensively).

In the fourth stage, the Party Panel results are compiled into three documents: the first is an infographic to provide feedback to the Party Panel participants; the second, a report for prevention organizations; and the third, a scientific article to spread the results globally. Although in all three, the Party Panel team makes an effort to utilize knowledge of the dynamics of behavior change to make sense of the results, this especially has an added value in the report for prevention organizations. In that report, the results regarding which determinants and sub-determinants appear the best intervention targets are interpreted and matched to behavior change methods to provide specific recommendations regarding promising prevention messages. This way, even prevention agencies that do not themselves have in-house behavior change expertise can readily use these results to guide their intervention development. The interventions and communications that are developed with this information can then be communicated to the target population using the Celebrate Safe network of ambassadors. 


\section{Logistics, workflow and Full Disclosure}

Party Panel was designed to require minimal funds to operate, using mainly free and open source software. Party Panel runs on a managed server in a Tier-3+ grade datacenter located in the Netherlands. This server runs a custom coded Party Panel website, as well as the questionnaire engine, the free and open source software LimeSurvey (LimeSurvey Project Team / Carsten Schmitz, 2017). To protect participants, all traffic uses the Hyper Text Transfer Protocol Secure (HTTPS).

LimeSurvey uses a MySQL and PHP backend, and the MySQL server is configured to only accept local traffic. In addition, no questions are asked that enable identifying the participants. This means that even if the server were to be compromised, no incriminating data can be seized. Finally, the data on the server are backed up every four hours and stored off-site for one month.

For analysis, the Party Panel data are downloaded locally and analyzed using R, RStudio, and RMarkdown (Allaire et al., 2017; R Development Core Team, 2017; RStudio Team, 2016; Xie, 2015).

The analysis script files evolve as the project progresses, so innovations retroactively and automatically apply to data from prior Party Panel rounds, and efficiency increases over the project lifespan. The project has a strong Full Disclosure character (Gjalt-Jorn Ygram Peters, Kok, Crutzen, \& Sanderman, 2017; G.-J.Y. Peters, Abraham, \& Crutzen, 2012), and all resources and data are made available at the latest two years after a Party Panel wave starts. This potential two-year embargo was implemented because Party Panel was designed to address topical issues, and therefore, press agencies may consider the results newsworthy. However, where it concerns nightlife-related risk behaviors, press often takes a sensationalist perspective, and the resulting misrepresentation of the situation may result in damaging government policies (e.g. more stringent and/or abstinence-based judicial measures or other policies). Therefore, despite the strong commitment to open science, it was deemed unethical to implement a workflow where data were made public immediately and automatically in all cases. The git protocol is used as version control system, using repositories that synchronize with GitHub. This GitHub repository is then synchronized to a dedicated component in the Open Science Framework project for Party Panel (https://osf.io/s4fmu). 


\section{The Party Panel questionnaire}

Ideally, a broad spectrum of determinants from different theories is measured (Gjalt-Jorn Ygram Peters \& Crutzen, 2017), but in the first waves, most potentially relevant beliefs identified in the second stage fit with a Reasoned Action Approach (Fishbein \& Ajzen, 2010). The questionnaires start with an informed consent, followed by questions about how frequently participants visited different types of nightlife venues (e.g. bars, clubs, and festivals). The questionnaires then ask some general introductory questions about the target behavior(s) and related behavior(s), after which the frequency and intensity with which participants engaged in the target behavior(s) are measured. In waves where multiple target behaviors are selected, participants are designated a random number, each random number corresponding to one of the target behaviors. Participants then receive an explanation about this system, and are asked whether they would be willing to answer questions about other target behaviors, as well. This method minimizes the participant burden of each participant while still enabling collecting data pertaining to multiple target behaviors.

The modifiable psychological determinants are then measured (for more background, see the section "Basics of behavior change" below). The variables at higher levels of psychological aggregation (i.e. the most "proximal" determinants, see Gjalt-Jorn Ygram Peters \& Crutzen, 2017) are measured first, and the variables at the lowest levels of psychological aggregation (i.e. the most specific determinants, such as beliefs) are measured last. For example, for RAA variables, the socalled 'direct' measures of each determinant precede the so-called 'indirect' or 'belief-based' measures. The reason for this order (i.e. ascending specificity) is that variables at higher levels of psychological aggregation may be seen as consisting of variables at lower levels of psychological aggregation. If the lower-level variables are measured first, they may still be salient once the higherlevel variables are measured, and this salience may influence information processing such that the measurement of the higher-level variables is biased, inducing artificially high correlations between higher-level variables and the lower-level variables they encompass. 


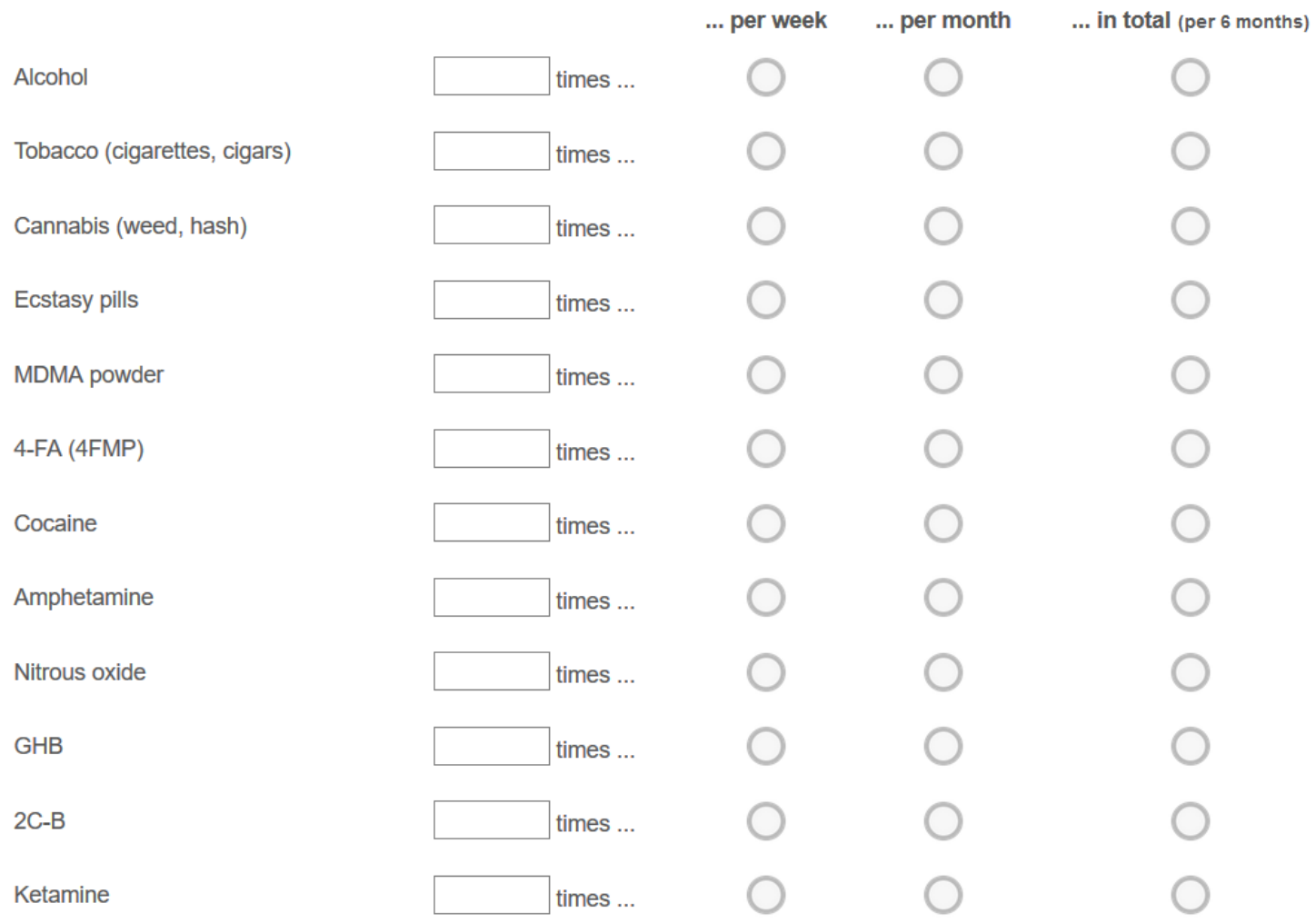

202

The second novel question type was a combination of two common question types. The first is the regular matrix-based Likert-scale question type. In this question format, participants are presented with a table of radio buttons corresponding to answer options. This table has a row and a column header, with the row header containing the subquestions for each row, and the column header containing the labels for the answer options in each column. A disadvantage of this question type is that all subquestions have to be crafted such that they fit naturally with the same set of answer options. This often leads to answer options being chosen that bear little meaning, instead being used 
as generic placeholders intended to correspond to different magnitudes. For example, "completely disagree" to "completely agree" is often used in combination with subquestions formulated as statements. For aspects that are not unequivocally unidimensional, this can create ambiguous situations. For example, to measure the belief whether a participant enjoy the hallucinogenic effects of MDMA (which are only experienced as higher doses), the subquestion "When I use MDMA, I enjoy experiencing hallucinogenic effects" could be used with answer options anchored by "completely disagree" versus "completely agree". Complete disagreement with this statement can mean that participants do not particularly care for hallucinogenic effects, but it can also mean that participants strongly dislike hallucinogenic effects. Using dedicated anchors, for example "very unpleasant" to distinguishing those answers. However, explicitly bidimensional anchors will often not apply equally well to all subquestions. A second question type, the semantic differential, resolves this: semantic differentials consist of several antonym pairs, introduced by the same lead-in (or stem). The second new question type was a combination of these two question types (Likert scales and semantic different anchors (see Figure 2).

First a number of questions about how for you, using a high dose of XTC differs from using a regular dose of XTC (MDMA).

If I use a high dose of XTC, my trip is ...
If I use a high dose of XTC, I get ...
If I use a high dose of XTC, I feel ...
If I use a high dose of XTC, time passes ...
If I use a high dose of XTC, I feel ...

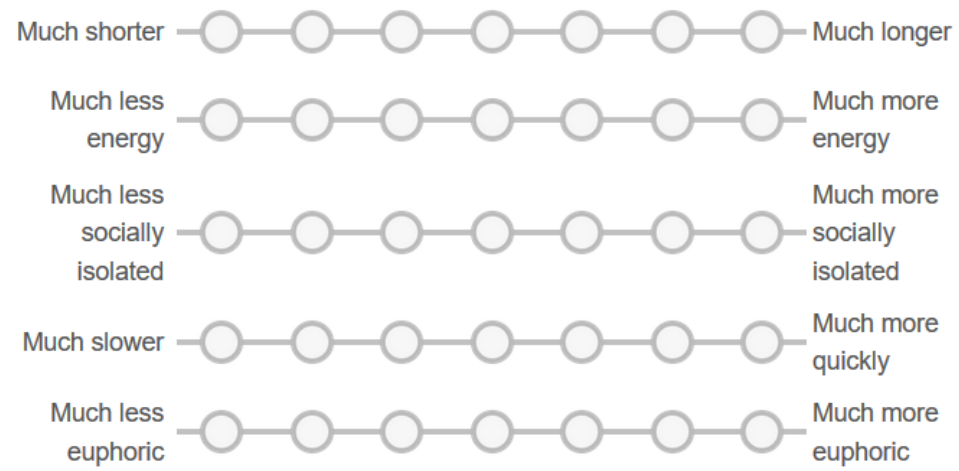



customization, we aimed to make it as easy as possible for other researchers to copy or adapt these question types. Finally, participants can optionally enter their 'Party Panel Code': a six-character code they can generate using their first name, last name, date of birth, place of birth, and eye color (see https://partypanel.eu/?code). This code was devised to enable following participants over successive Party panel waves while securing their anonymity. After the first Party Panel wave, it became clear that a large proportion of participants used mobile devices (e.g. mobile phones) to complete the Panel repository at the Open Science Framework).

\section{Basics of behavior change: determinants and behavior change principles}

The data collected with each Party Panel wave are then analysed using the R Markdown scripts 
outcomes. In psychological research, cortical firing patterns are often not studied at the neuronal level, but at a much higher level, where psychological constructs are hypothesized as metaphors for aspects of the human psychology. Such constructs can be considered as a type of 'variables' that can then be measured and manipulated, such as an individual's degree of extraversion, optimism, intention to moderate their drinking, or their attitude about using a high dose of ecstasy. From this point of view, then, behavior change requires first obtaining as complete a picture of the relevant aspects of individuals' psychology, and second, effectively prompting learning in the target individuals such that these relevant aspects of their psychology align with the desirable behavior.

In behavior change science, psychological constructs that predict human behavior are commonly called 'determinants'. Identifying the important determinants for a target behavior, then, is the first prerequisite task before it is possible to productively think about intervention development (Crutzen et al., 2017; Gjalt-Jorn Ygram Peters \& Crutzen, 2017, 2018; G.-J.Y. Peters, 2014). A number of well-known behavioral determinants are attitude (the subset of an individuals' representations of the world that relates to perceived consequences of the target behavior and the individuals' evaluation of those consequences in terms of desirability), perceived norms (the perceived approval or disapproval of important social referents, as well as the perceived behavior of important social referents), and perceived behavioral control (an individual's perceived capability to perform the target behavior as well as perceptions relating to barriers and facilitating conditions in the environment). Psychological theories have defined hundreds of such determinants, often with overlapping definitions and operationalisations (Gjalt-Jorn Ygram Peters \& Crutzen, 2017). These determinants are defined at a very generic level, because they are studied for human behavior in general. Changing a specific target behavior in a specific target population, therefore, always requires identifying the important subdeterminants underlying those determinants. For example, individuals' attitudes regarding using a high dose of MDMA depend in part on whether they believe that a high dose increases the euphoric effects, while their attitude regarding using hearing protection depend in part on whether they 
believe that ear plugs distort the music. These subdeterminants are what are targeted by the messages in behavior change interventions.

Once the most important determinants and subdeterminants have been identified, the second task is matching these determinants to behavior change principles that engage relevant evolutionary learning processes and thereby prompt learning in the target individuals (Crutzen \& Peters, 2018).

Different types of behavioral determinants relate to different parts of the human psychology, and thereby, different parts and sometimes types of memory, such as procedural, declarative, and episodic (or autobiographical) memory. Because of this, there exist no behavior change principles that can always be relied on to change any (sub-)determinant. Instead, different behavior change principles are effective for changing different (sub-)determinants. Once a list of important subdeterminants has been obtained, knowing which determinants they are a part of enables inspecting the psychological literature to identify the most effective behavior change principles to target those (sub-)determinants. These can then be translated to practical applications that can be integrated into behavior change interventions (Kok, 2014; Kok et al., 2016; Gjalt-Jorn Ygram Peters \& Kok, 2016).

Thus, effective prevention requires two types of research. On the one hand, it is important that trends in behavior and behavioral patterns are followed, such as trends in substance use. Which substances are popular? Which poly-drug combinations are becoming more prevalent? Which harm reduction strategies are employed by users? Once such patterns have been identified, it becomes clear where to focus prevention efforts. Then, to inform those prevention efforts, the determinants and subdeterminants underlying those behaviors must be identified. This need is fulfilled by Party Panel.

\section{Conclusion}

The architecture of the Party Panel lifecycle is relatively cheap and can feasibly be translated into different contexts and countries. It offers a lean workflow to effectively combine the expertise of different parties to realize effective health promotion in nightlife or other settings. By combining such 
research efforts with monitoring of drug markets and the specific behavioral patterns of substance users, it becomes possible to swiftly and effectively intervene to reduce the harms associated with substance use.

\section{References}

Allaire, J. J., Cheng, J., Xie, Y., McPherson, J., Chang, W., Allen, J., ... Arslan, R. (2017). rmarkdown: Dynamic Documents for R.

Bartholomew Eldredge, L. K., Markham, C. M., Ruiter, R. A. C., Fernández, M. E., Kok, G., \& Parcel, G. S. (2016). Planning Health Promotion Programs: an Intervention Mapping Approach (4th ed.). San Francisco, CA: Jossey-Bass.

Bellis, M. A., Hughes, K., McVeigh, J., Thomson, R., \& Luke, C. (2005). Effects of nightlife activity on health. Nursing Standard, 19(30), 63-71.

Calafat, A., \& Juan, M. (2004). Health and safety problems in recreational nightlife in the Island of Mallorca. International Journal of Drug Policy, 15, 157-162.

Crutzen, R., \& Peters, G.-J. Y. (2018). Evolutionary learning processes as the foundation for behaviour change. Health Psychology Review, 12(1), 43-57. https://doi.org/10.1080/17437199.2017.1362569

Crutzen, R., Peters, G.-J. Y., \& Noijen, J. (2017). Using Confidence Interval-Based Estimation of Relevance to select social-cognitive determinants for behaviour change interventions. Frontiers in Public Health. https://doi.org/10.3389/fpubh.2017.00165

Eker, H. H., Lieshout, E. M. M. Van, Hartog, D. Den, \& Schipper, I. B. (2010). Trauma Mechanisms and Injuries Associated with Go-Karting, 107-110.

Fishbein, M., \& Ajzen, I. (2010). Predicting and changing behavior: The reasoned action approach. New York: Psychology Press.

Glanz, K., \& Bishop, D. B. (2010). The role of behavioral science theory in development and implementation of public health interventions. Annual Review of Public Health, 31, 399-418. 
Hu, F. B., Li, T. Y., \& Colditz, G. A. (2003). Television Watching and Other Sedentary Behaviors in Relation to Risk of Obesity and Type 2 Diabetes Mellitus in Women. JAMA, 289(14), 17851791.

Hughes, K., Anderson, Z., Morleo, M., \& Bellis, M. A. (2007). Alcohol , nightlife and violence : the relative contributions of drinking before and during nights out to negative health and criminal justice outcomes, 60-65. https://doi.org/10.1111/j.1360-0443.2007.02030.x

Kok, G. (2014). A practical guide to effective behavior change: How to apply theory- and evidencebased behavior change methods in an intervention. European Health Psychologist, 16(5), 156170.

Kok, G. (2016). Social Psychology Applied: Politics, Theories, and the Future. Maastricht, the Netherlands: Maastricht University.

Kok, G., Gottlieb, N. H., Peters, G.-J. Y., Mullen, P. D., Parcel, G. S., Ruiter, R. A. C., ... BartholomewEldredge, L. K. (2016). A taxonomy of behavior change methods: an Intervention Mapping approach. Health Psychology Review, 10(3). https://doi.org/10.1080/17437199.2015.1077155

LimeSurvey Project Team / Carsten Schmitz. (2017). LimeSurvey: An Open Source survey tool. Hamburg, Germany.

Martinez-Gonzalez, M. A., Alfredo Martinez, J., Hu, F. B., Gibney, M. J., \& Kearney, J. (1999). Physical inactivity, sedentary lifestyle and obesity in the European Union. International Journal of Obesity, 23, 1191-1201.

Masters, R., Anwar, E., Collins, B., Cookson, R., \& Capewell, S. (2017). Return on investment of public health interventions: a systematic review. Journal of Epidemiology \& Community Health, 1-8. https://doi.org/10.1136/jech-2016-208141

Montain, S. J., Sawka, M. N., \& Wenger, C. B. (2001). Hyponatremia Associated With Exercise : Risk Factors and Pathogenesis. Exercise and Sport Sciences Reviews, 29(3), 113-117. 
Nabben, T., Luijk, S. J., Benschop, A., \& Korf, D. J. (2017). Antenne 2016: trends in alcohol, tabak en drugs bij jonge Amsterdammers [Antenna 2016: Alcohol, tobacco and drug trends among Amsterdam adolescents and young adults]. Amsterdam: Rozenberg Publishers.

Peters, Gjalt-Jorn Ygram, \& Crutzen, R. (2017). Pragmatic Nihilism: How a Theory of Nothing can help health psychology progress. Health Psychology Review, 11(2), 103-121. https://doi.org/10.1080/17437199.2017.1284015

Peters, Gjalt-Jorn Ygram, \& Crutzen, R. (2018). Establishing determinant importance using CIBER: an introduction and tutorial. European Health Psychologist, 20(3), 485-495. https://doi.org/10.31234/osf.io/5wjy4

Peters, Gjalt-Jorn Ygram, \& Kok, G. (2016). All models are wrong, but some are useful: A comment on Ogden (2016). Health Psychology Review, 10(3), 265-268. https://doi.org/10.1080/17437199.2016.1190658

Peters, Gjalt-Jorn Ygram, Kok, G., Crutzen, R., \& Sanderman, R. (2017). Health Psychology Bulletin: improving publication practices to accelerate scientific progress. Health Psychology Bulletin, 1(1), 1-6. https://doi.org/10.5334/hpb.2

Peters, Gjalt-Jorn Ygram, Ruiter, R. A. C., \& Kok, G. (2014). Threatening communication: A qualitative study of fear appeal effectiveness beliefs among intervention developers, policymakers, politicians, scientists and advertising professionals. International Journal of Psychology, 49(2), 71-79. https://doi.org/10.1002/ijop.12000

Peters, G.-J.Y. (2014). A practical guide to effective behavior change: how to identify what to change in the first place. The European Health Psychologist, 16, 142-155.

Peters, G.-J.Y., Abraham, C., \& Crutzen, R. (2012). Full disclosure: doing behavioural science necessitates sharing. The European Health Psychologist, 14, 77-84.

R Development Core Team. (2017). R: A Language and Environment for Statistical Computing. Vienna, Austria.

RStudio Team. (2016). RStudio: Integrated Development Environment for R. Boston, MA. 
Schaafsma, D., Stoffelen, J. M. T., Kok, G., \& Curfs, L. M. G. (2013). Exploring the development of existing sex education programmes for people with intellectual disabilities: an intervention mapping approach. Journal of Applied Research in Intellectual Disabilities : JARID, 26(2), 157-166. https://doi.org/10.1111/jar.12017

Tinsley, H. E. A., \& Eldredge, B. D. (1995). Psychological Benefits of Leisure Participation: A Taxonomy of Leisure Activities Based on Their Need-Gratifying Properties. Journal of Counseling Psychology, 42(2), 123-132.

Van Laar, M. W., Van Ooyen-Houben, M. M. J., Cruts, A. A. N., Meijer, R. F., Croes, E. A., Ketelaars, A. P. M., \& van der Pol, P. M. (2016). Nationale Drug Monitor: jaarbericht 2016. Utrecht/Den Haag.

van Liempt, I. (2015). Safe nightlife collaborations: Multiple actors, conflicting interests and different power distributions. Urban Studies, 52(3), 486-500. https://doi.org/10.1177/0042098013504010 Xie, Y. (2015). Dynamic Documents with R and knitr (2nd ed.). Chapman and Hall/CRC. 